\title{
Green Synthesis of Gold Nanoparticles Using Glycerol as a Reducing Agent
}

\author{
Pradnya Nalawade, Tulsi Mukherjee, Sudhir Kapoor \\ Radiation \& Photochemistry Division, Bhabha Atomic Research Centre, Mumbai, India \\ Email: "sudhirk@barc.gov.in
}

Received December 27, 2012; revised January 29, 2013; accepted February 5, 2013

Copyright (C) 2013 Pradnya Nalawade et al. This is an open access article distributed under the Creative Commons Attribution License, which permits unrestricted use, distribution, and reproduction in any medium, provided the original work is properly cited.

\begin{abstract}
We report one pot synthesis of uniform and stable polyvinyl pyrolidone (PVP) protected gold nanoparticles (Au NPs) using environmental friendly, glycerol as reducing agent. The effect of the presence of a capping agent (PVP) and the concentration of reactants (glycerol, tetra chloroauric acid, and $\mathrm{NaOH}$ ) on the size and homogeneity of the Au NPs formed were investigated. Highly stable and well-distributed Au NPs were obtained at higher concentration of $\mathrm{NaOH}$ in the presence of PVP with a clear dependence of the size and the concentration of glycerol, $\mathrm{NaOH}$ and the presence of capping agent, whereas, large heterogeneous Au NPs were obtained in absence of PVP. The particle morphology, size and crystallinity were characterized using UV-Vis spectroscopy, transmission electron microscopy and X-ray diffraction techniques. The catalytic performance of as synthesized Au NPs for the reduction of o-nitro aniline was investigated in aqueous solution. The pseudo-first-order rate constants were also calculated for the catalytic reaction.
\end{abstract}

Keywords: Gold Nanoparticles; Glycerol; Synthesis; Catalytic Reaction; Characterization

\section{Introduction}

In recent decades there in growing interest in the synthesis of metal nanoparticles due to their unique properties, which are significantly different from the behavior of the respective bulk material [1,2]. Gold nanoparticles (Au NPs) have wide attraction because of their electronic, biosensing, plasmonic, photonics, catalysis, biomedical and surface-enhanced Raman scattering (SERS) properties [2-7]. Most of the chemical methods reported in the literature for the synthesis of Au NPs often involve use of toxic reducing agents (such as sodium borohydride, hydrazine, etc.) and harsh reaction parameters like high temperatures in the polyol method [8-10]. Glycerol, also known as glycerin, is commonly used in the pharmacological application and its derivative used in the synthesis of drugs [11]. It is also used as a sweetener in food industry and due to its hygroscopic nature it is widely used as dehydrating and moistening agent [12]. Besides that it is easily biodegradable in aerobic conditions thus can be replaced by traditional reducing agent.

Few studies have been reported in the literature demonstrating the formation of silver and Au NPs using glyc-

*Corresponding author. erol as reducing agent at low temperature. Genc et al. have shown low temperature method to obtain mo- nodispersed $\mathrm{Au}$ NPs using glycerol-incorporated nanosized liposome, where glycerol, is incorporated on both the external and internal polar surfaces of liposome encapsulating chloroauric acid, facilitates the reduction of $\mathrm{Au}(\mathrm{III})$ to form $\mathrm{Au}(0)$ atoms and subsequently nanoparticles [13]. Singh et al. have reported the formation of nickel nanoparticles in glycerol at $100^{\circ} \mathrm{C}$ by using hydrazine hydrate in alkaline medium [14]. Nisaratanaporn and Wongsuwan prepared silver powders of par- ticle size more than $63 \mathrm{~nm}$ using silver alkoxide as sil- ver ion precursor and glycerol as a reducing agent at high temperature [15]. Grace and Pandian reported the synthesis of spherical and prism shaped Au NPs in gly- cerol at both reflux and microwave conditions [16].

As water is the most beneficial solvent it will be of interest to study the effect of water in glycerol on the formation of NPs. To the best of our knowledge, there is no report demonstrating the effect of concentration of glycerol, $\mathrm{NaOH}$ and capping agent on the formation of $\mathrm{Au}$ NPs at room temperature without requiring any additional reactants. In this paper we report environmentally friendly, low temperature method to prepare $\mathrm{Au}$ NPs without using any external reducing agent. The 
formed particles were further studied for their catalytic application in reduction of o-nitro aniline.

\section{Materials and Methods}

\subsection{Materials}

Tetra chloroauric acid, glycerol, PVP (Mol. wt. 40000), sodium hydroxide, o-nitro aniline and sodium borohydride were purchased from Sigma-Aldrich and used as received. Millipore purified water having 18.2 $\mathrm{M} \Omega$ electrical resistivity was used for making solutions.

\subsection{Characterization}

Absorption measurements were carried out on a Jasco V650 spectrophotometer. The spectra were recorded at room temperature using $1 \mathrm{~cm}$ quartz cuvette. Samples for transmission electron microscopy (TEM) were prepared by putting a drop of the colloidal solution on a copper grid coated with a thin amorphous carbon film placed on a filter paper. The excess solvent was removed using a filter paper, and letting the solvent evaporate at room temperature. TEM characterization was carried out using a Phillips CM 200 electron microscope with working voltage $200 \mathrm{kV}$ having magnification 34,000 to 78,000 . Particle size was measured by TEM photograph and calculated the size by considering at least 100 particles. XRD measurement was carried out on precipitated nanoparticles using Philips X'pert Pro machine with monochromatised $\mathrm{CuK} \alpha \mathrm{X}$-ray source operated at $20 \mathrm{kV}$ and $30 \mathrm{~mA}$.

\subsection{Method}

\subsubsection{Synthesis of Au NPs in Glycerol or Water: Glycerol System}

All the solutions were prepared freshly in order to avoid any photochemical reactions and experiments were carried out in an aerated condition. Gold sol was prepared by mixing the required concentration of $\mathrm{NaOH}\left(5 \times 10^{-4}\right.$ $\left.\mathrm{M}-1 \times 10^{-3} \mathrm{M}\right)$ and chloroauric acid solution $\left(1 \times 10^{-4}\right.$ $\left.\mathrm{M}-1 \times 10^{-3} \mathrm{M}\right)$ in either neat glycerol or glycerol-water mixtures [80 to $20 \%(\mathrm{v} / \mathrm{v})$ glycerol in water] at room temperature in the absence or presence of PVP $[0.05 \%$ $0.1 \%(w / v)]$. Gradual formation of different color with time indicates the formation of Au NPs (Scheme 1). The experiments were repeated at least three times and found to be within the experimental error of $\pm 5 \%$.

\subsubsection{Catalytic Reduction of o-Nitro Aniline}

The catalytic reduction reaction was carried out in $5 \mathrm{ml}$ volumetric flask. In a typical reaction excess of ice cold $\mathrm{NaBH}_{4}\left(3 \times 10^{-2} \mathrm{M}\right)$ was mixed with o-nitro aniline $(2.3$ $\times 10^{-4} \mathrm{M}$ ) solution in water. This was followed by the addition of Au NPs. The absorption spectra were recorded immediately after mixing with a time interval of 2 min in a scanning range of $200-600 \mathrm{~nm}$ at $25^{\circ} \mathrm{C}$ till the yellow colored o-nitro aniline solution became colorless (from 4 - 25 min depending upon the concentration of catalyst used). The experiments were repeated at least three times and found to be within the experimental error of $\pm 10 \%$.

\section{Results and Discussion}

\subsection{Formation Au NPs in Neat Glycerol}

Metal nanoparticles (especially $\mathrm{Ag}, \mathrm{Au}$ and $\mathrm{Cu}$ ) exhibit a unique UV-Vis absorption band derived from collective oscillation of conduction electrons upon interaction with electromagnetic radiation, known as surface plasmon resonance (SPR) [2]. The absorption maximum of SPR depends on the shape and size of the particles [2]. This optical property of noble metal NPs is exploited for many applications [2]. In the present work Au NPs were prepared by simply adding $\mathrm{NaOH}$ to the tetra chloroauric acid solution in neat glycerol. Formation of nanoparticles
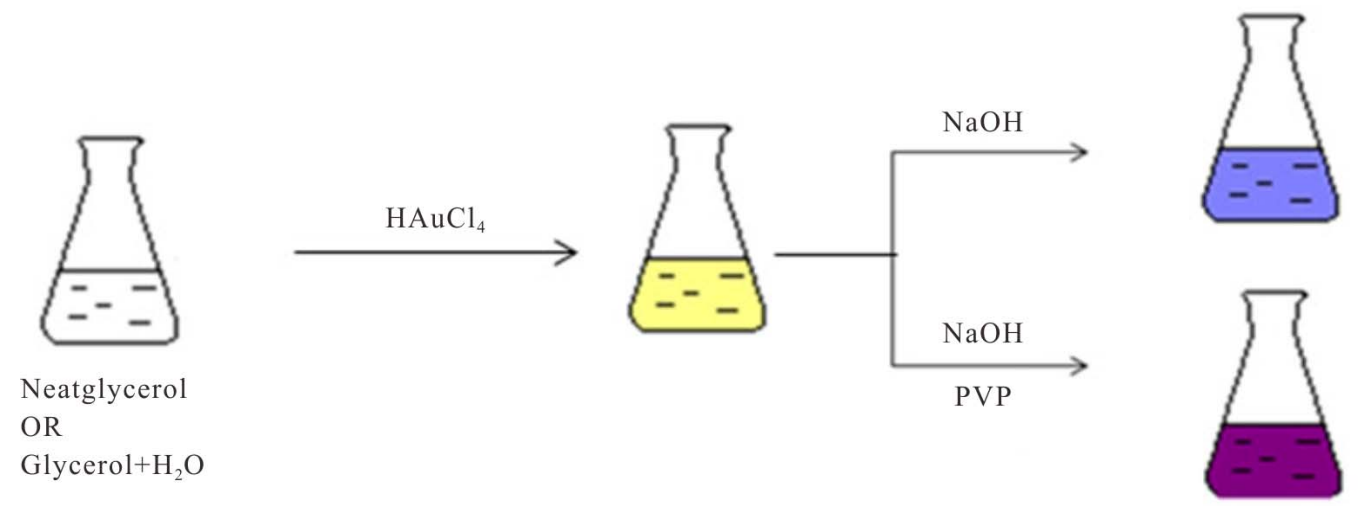

Gold nanoparticles

Scheme 1. Synthesis of gold nanoparticles. 
was observed, after 2 min of shaking, by change in the color of the solution from light yellow to colorless to pinkish violet. Figure 1 shows the typical changes in absorption UV-Vis spectra of the Au NPs in neat glycerol. The initial peaks at $505 \mathrm{~nm}$ due to the surface plasmon resonance of Au NPs which is red shifted up to 536 $\mathrm{nm}$ along with formation of new band at longer wavelength $(748 \mathrm{~nm})$ with time, this might be due to formation of the anisotropic Au NPs which always exhibit two to three SPR bands depending on their shape compared with single SPR for small spherical nanocrystals [17]. As can be seen from the TEM image of the Au NPs, prepared in neat glycerol (Figure 1(b)), that the formed par-
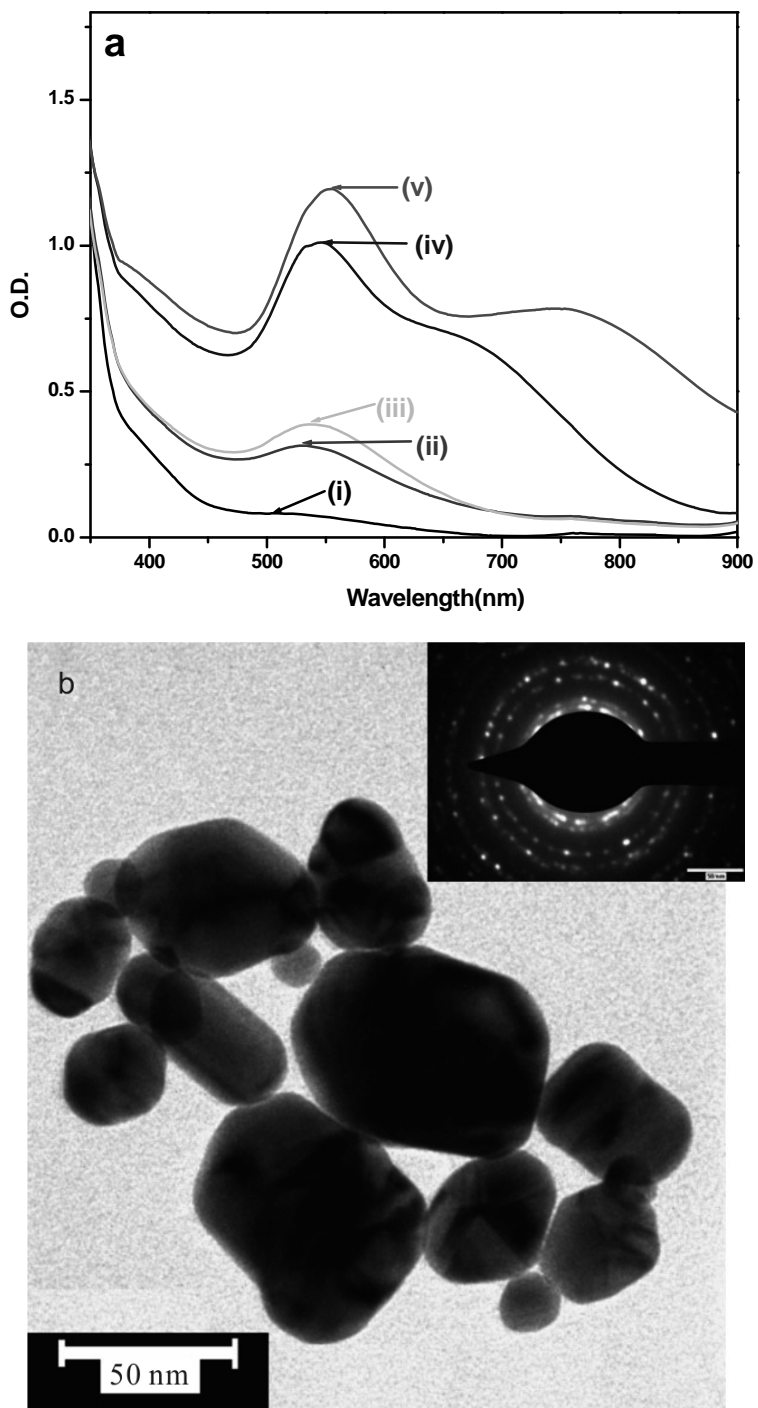

Figure 1. (a) Variation in the surface plasmon absorption band of $\mathrm{Au}$ NPs formed on mixing $5 \times 10^{-4} \mathrm{M} \mathrm{HAuCl}_{4}$ and 1 $\times 10^{-3} \mathrm{M} \mathrm{NaOH}$ in neat glycerol (i) after 3 min (ii) after 5 min (iii) after $48 \mathrm{~min}$ (iv) after $24 \mathrm{hr}$ (v) after 6 days; (b) Corresponding TEM image and electron diffraction pattern.

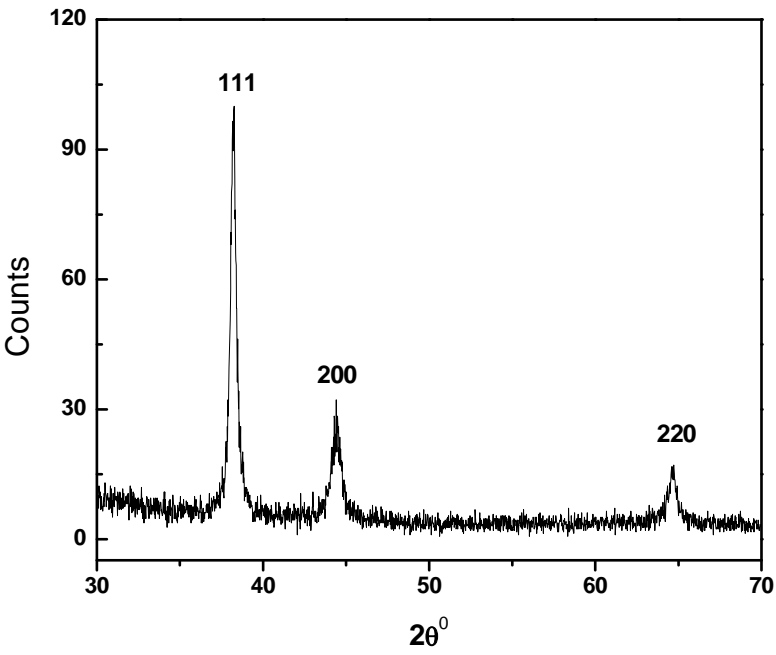

Figure 2. XRD pattern of Au NPs.

ticles are nonspherical including nanorod with average particle size $30 \mathrm{~nm}$. This non spherical nature of the nanoparticles caused the absorption at longer wavelength in absence of stabilizing agent. Yang et al. have shown the formation of anisotropic Au NPs of average diameter of $50-100 \mathrm{~nm}$ without using any stabilizing agent [18]. The colloidal stability of particles prepared in neat glycerol was not good as they starts to aggregate after about 24 hrs. Figure 2 shows the XRD pattern in the $2 \theta$ range $30^{\circ}-70^{\circ}$ for Au NPs prepared in neat glycerol. The patterns exhibit peaks due to diffraction from (111), (200) and (220) planes of metallic gold (JCPDS No. 04-0784). The XRD pattern suggests the formation of crystalline Au NPs with face-centred cubic (fcc) structure. Broadening of XRD peaks clearly indicate that the samples are nanocrystalline in nature. The particle size (d) was also calculated from XRD line broadening data, after instrumental line broadening correction, using Scherrer formula, $k \lambda=\mathrm{d} \cdot \beta \cdot \cos \theta$, where $\lambda$ is wavelength of the X-ray used, and it is $1.5406 \AA, \beta$ is the angular line width at half maximum intensity, $\theta$ is Bragg diffraction angle and $k$ is a constant, and is equal to 0.9 [19]. The particle size calculated from Figure 2, was found to be $\sim 25 \mathrm{~nm}$.

\subsection{Effect of PVP Concentration}

Figure 3 also shows the effect of concentrations of PVP on UV-Vis optical absorption spectra of the Au NPs sols prepared by addition of $1 \times 10^{-3} \mathrm{M} \mathrm{NaOH}$ and $5 \times 10^{-4}$ $\mathrm{M}$ tetra chloroauric acid in neat glycerol. PVP was found to be a very efficient stabilizer for the stabilization of $\mathrm{Au}$ NPs. Even a small concentration of PVP $(0.05 \%)$ was sufficient to stabilize the Au NPs. When concentration of PVP decreased to $0.01 \%$ the red shift of absorption maxima was observed which might be due to the increase 
in particle size of the Au NPs. However, concentration of PVP $(\geq 0.05 \%)$ has no significant effect on stability as well as size, as Au NPs prepared with different concentrations are equally stable for more than a year. Since the particles were stable in the sol form XRD could not be carried out, nevertheless, TEM and selective area electron diffraction (SAED) confirmed the presence of fcc Au NPs (results not shown).

\subsection{Effect of $\mathrm{NaOH}$ Concentration}

The above results showed that concentration of PVP ( $\geq$ $0.05 \%$ ) had no significant effect on the stability of the particles; hence, for the preparation of particles we had selected $0.1 \%$ PVP to make sure that the particles do not aggregate. Figure 4 shows the effect of $\mathrm{NaOH}$ concentration $\left(5 \times 10^{-4} \mathrm{M}-1 \times 10^{-3} \mathrm{M}\right)$ on the UV-Vis absorption spectrum of $\mathrm{Au}$ NPs in presence of $0.1 \%$ PVP in neat glycerol. The blue shift of SPR band with increasing concentration of $\mathrm{NaOH}$ indicates the decrease in particle size of Au NPs. The corresponding TEM image of Au NPs formed in presence of $5 \times 10^{-4} \mathrm{M}-1 \times 10^{-3}$ $\mathrm{M} \mathrm{NaOH}$ (Figures 5(a) and (b)) shows that the average particle size falls from $20 \mathrm{~nm}$ to $5-7 \mathrm{~nm}$ when the $\mathrm{NaOH}$ concentration was increased from $5 \times 10^{-4} \mathrm{M}-1$ $\times 10^{-3} \mathrm{M} \mathrm{NaOH}$. Since the particles were stable in the sol form XRD could not be carried out. Chen et al. have observed similar results where palladium nanoparticles in the size range of 8.6 to $2.4 \mathrm{~nm}$ were prepared by changing the concentration of $\mathrm{NaOH}$ in presence of PVP [20]. However, in our case, no particle formation was observed when concentration of $\mathrm{NaOH}$ was below $5 \times$ $10^{-4} \mathrm{M}$.

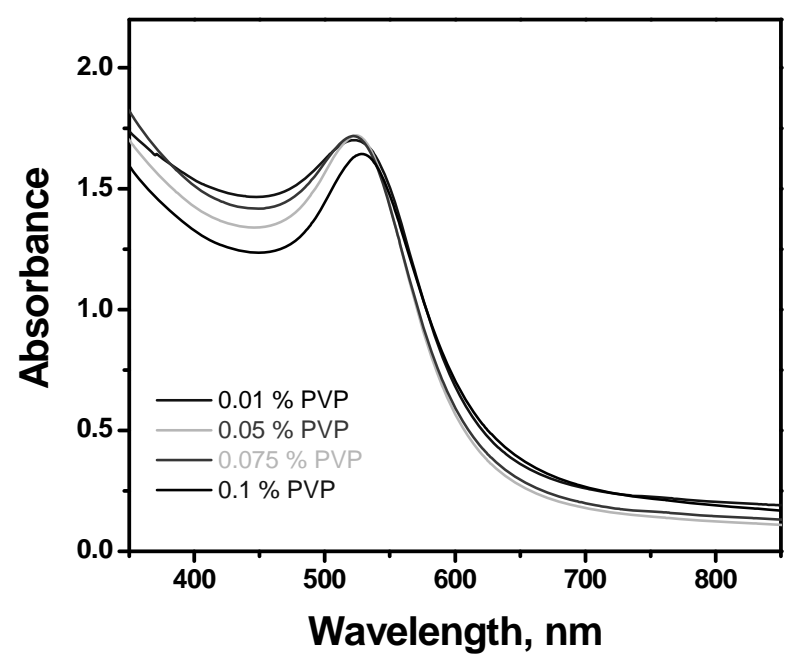

Figure 3. Effect of PVP concentration (wt \%) on the optical absorption spectrum of Au NPs prepared by addition of $1 \times$ $10^{-3} \mathrm{M} \mathrm{NaOH}$ and of $5 \times 10^{-4} \mathrm{M}$ tetra chloroauric acid. (i) 0.01 (ii) 0.05 (iii) 0.075 and (iv) 0.1 . Spectra were recorded after $48 \mathrm{hr}$.

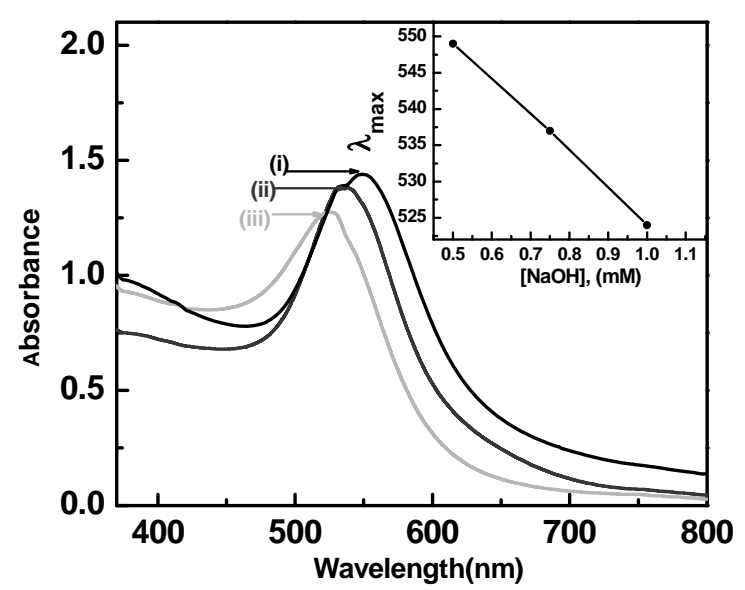

Figure 4. Effect of $\mathrm{NaOH}$ concentration on Au NPs sol synthesized by the reaction of (i) $5 \times 10^{-4}$, (ii) $7.5 \times 10^{-4}$ and (iii) $1 \times 10^{-3} \mathrm{M} \mathrm{NaOH}$ with $5 \times 10^{-4} \mathrm{M}$ auric acid in the presence of $0.1 \%$ PVP in glycerol.

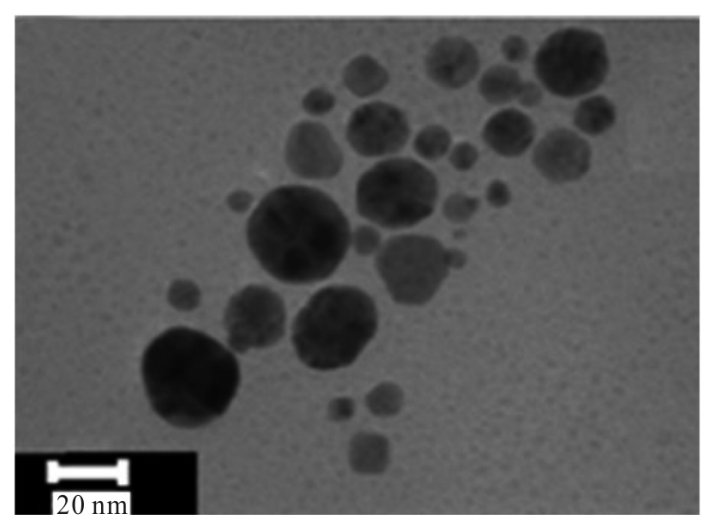

(a)

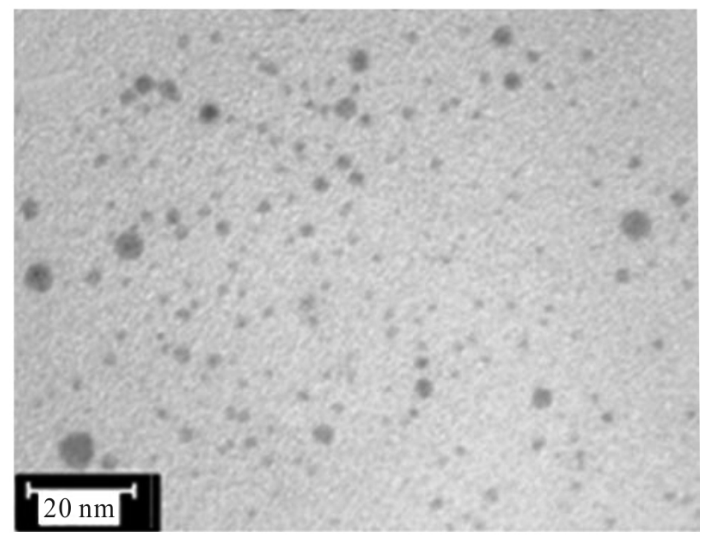

(b)

Figure 5. TEM image of Au NPs obtained with different concentration of $\mathrm{NaOH}$ other conditions are same as Figure 4. (a) $7.5 \times 10^{-4} \mathrm{M}$ (b) $1 \times 10^{-3} \mathrm{M}$.

\subsection{Effect of Gold Ion Concentration}

On addition of $\mathrm{NaOH}\left(1 \times 10^{-3} \mathrm{M}\right)$ to different concentrations of the tetra chloroauric acid solution in absence 
of PVP, the color of the solution changed from light yellow to colorless to shades of blue and violet. However, in presence of PVP the color of the gold sol range from wine red to pink to magenta to violet for gold ion concentration of $1 \times 10^{-4} \mathrm{M}, 2 \times 10^{-4} \mathrm{M}, 5 \times 10^{-4} \mathrm{M}$ and 7.5 $\times 10^{-4} \mathrm{M}$, respectively. Figure 6 shows the UV-Vis spectra of the Au NPs obtained for various concentrations of gold ions in the absence and presence of $0.1 \%$ PVP, at room temperature. The peak at $\sim 530 \mathrm{~nm}$ corresponds to the surface plasmon absorption of Au NPs. The absorption peak intensity of Au NPs in both absence and presence of PVP was found to increase and the $\lambda_{\max }$ gradually shifted towards higher wavelength with the increasing concentration of gold ions, this is possibly due to the formation of large size spherical nanoparticles at higher concentrations of gold ions. However, for the gold ions concentration of $5 \times 10^{-4} \mathrm{M}$ and above in absence of PVP,
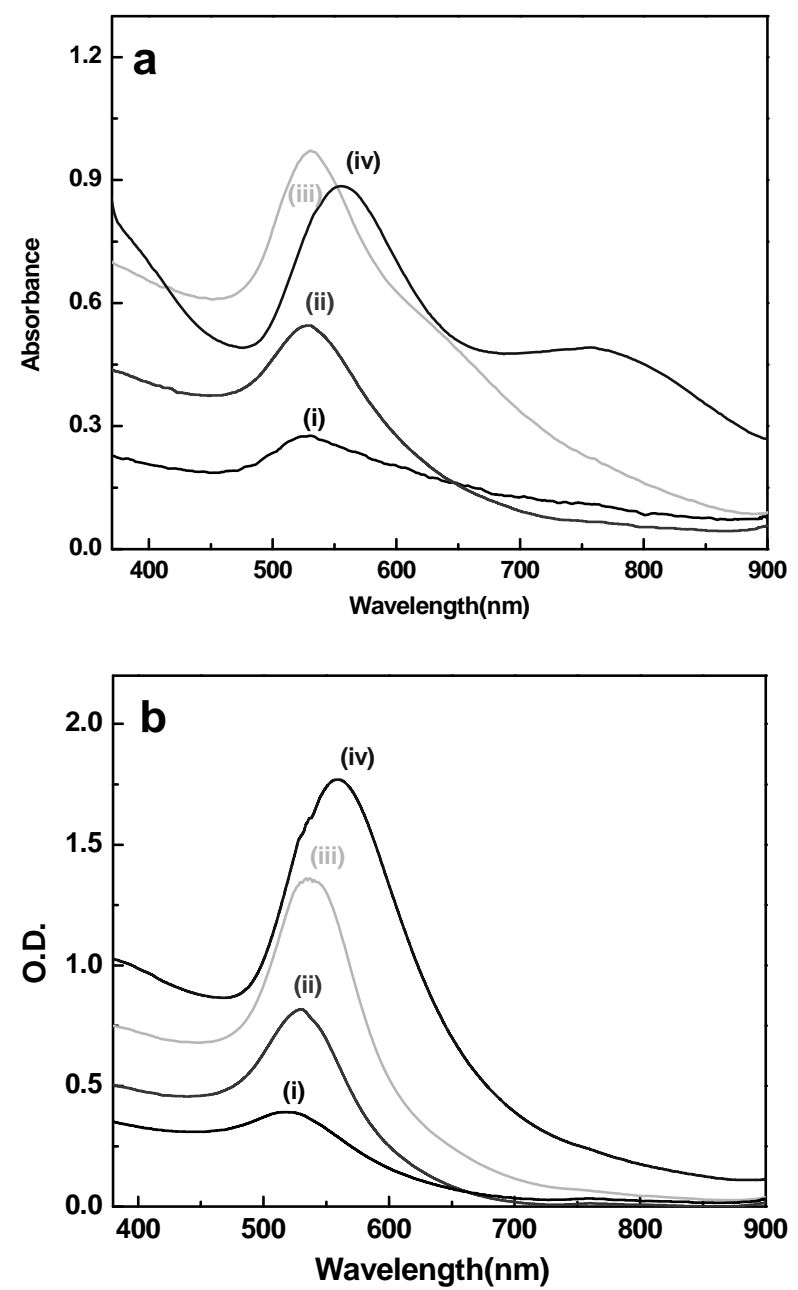

Figure 6. UV-Vis spectra showing effect of tetra chloroauric acid concentration on Au NPs sol synthesized by the reaction of (i) $1 \times 10^{-4}$, (ii) $2 \times 10^{-4}$, (iii) $5 \times 10^{-4}$ and (iii) $7.5 \times$ $10^{-4} \mathrm{M}$ auric acid with $5 \times 10^{-4} \mathrm{M} \mathrm{NaOH}$ in glycerol. (a) without PVP (b) with $0.1 \%$ PVP. All spectra were taken after $24 \mathrm{hr}$. the shoulder at $635 \mathrm{~nm}$ in the case of $5 \times 10^{-4} \mathrm{M}$ or new peak at $735 \mathrm{~nm}$ in the case of $7.5 \times 10^{-4} \mathrm{M}$ was observed which is possibly due to formation of nonsherical Au NPs as explained in Figure 1. Similar results were obtained by S. Boopathi et al. in the synthesis of Au NPs using tetraphenylborate and PVP for colorimetric detection of mercury ions [21]. Oza et al. studied the effect of chloroauric acid concentration on the formation of gold nanorods in presence of cetyl trimethylammonium bromide (CTAB) and it was found that high yield of nanorods formation occurred when the concentration of gold ions was $\geq 1 \times$ $10^{-3} \mathrm{M}$, whereas, yield of spherical Au NPs was high at lower concentration of gold ions [22]. However, in our study it was found that, on addition of stabilizing agent PVP during the synthesis, the band at longer wavelength was not observed indicating formation of almost spherical nanoparticles. This might be due to the combine effect of viscosity of medium and adsorption of PVP on the surface of nanoparticles during growth which result in to the formation of spherical nanoparticles. The increased absorbance may be correlated to the production of metal nanoparticles in larger quantities in the presence of higher concentration of gold ion precursor. It was also seen that at concentration of $5 \times 10^{-4} \mathrm{M}$ and $7 \times 10^{-4} \mathrm{M}$ tetra chloroauric acid more stable, spherical and small size Au NPs were formed. Also, the yield of the Au NPs was reasonably good. At both the concentrations similar size of particles $(\sim 10 \mathrm{~nm})$ were observed (results not shown). As mentioned above due to the good stability of the particles XRD could not be carried out.

Based on above results to get the good dispersion as well as stable Au NPs in neat glycerol the optimum concentrations of tetra chloroauric acid, $\mathrm{NaOH}$ and PVP are $5 \times 10^{-4} \mathrm{M}, 1 \times 10^{-3} \mathrm{M}$ and $0.1 \%(\mathrm{w} / \mathrm{v})$, respectively.

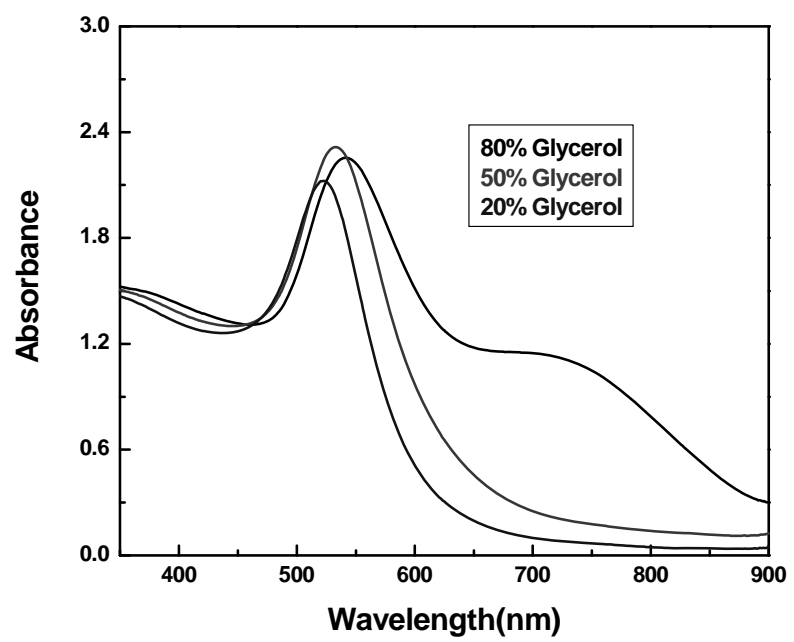

Figure 7. Effect of glycerol concentration on the UV-Vis spectra of $\mathrm{Au}$ NPs in presence of $1 \times 10^{-3} \mathrm{M} \mathrm{NaOH}$ and $7 \times$ $10^{-4} \mathrm{M} \mathrm{HAuCl}_{4}$ without using PVP. 


\subsection{Synthesis of Au NPs in Glycerol: Water Mixture}

To see the effect of concentration of glycerol on the particle size and shape of nanoparticles, synthesis of Au NPs were carried out at different concentrations of glycerol in water with fixed concentration of $\mathrm{NaOH}$ without using PVP. Figure 7 shows the UV-Vis spectra of the Au NPs obtained with different concentration ( $\mathrm{v} / \mathrm{v})$ of glycerol. In $80 \%$ glycerol two SPR band centered at 543 and 694 due of Au NPs was observed. This new band formation at longer wavelength indicates the presence of nonspherical nanoparticles along with spherical NPs. However when concentration of glycerol decreased up to $20 \%$, almost spherical and monodispersed nanoparticles were obtained. Figure 8 shows the TEM images of Au NPs obtained at $80 \%, 50 \%$ and $20 \%$ of glycerol. The shape and average particle size obtained by TEM measurements at different concentration of glycerol is summarized in Table $\mathbf{1}$.

The above results show that at low concentrations of glycerol it is possible to stabilize the particles without using stabilizer. It seems that the stability of the particles depend on the viscosity of the medium though not in direct proportionality. High viscosity means that formation of nuclei in a smaller zone and thus the interaction of particles in a smaller confined volume results in aggregation of the particles. At appropriate viscosity the interaction between the particles decreases and this leads to the stability of the particles. Thus, it can be concluded that for the preparation of small and stable Au NPs the mixture of glycerol:water is better as compared to neat glycerol.

\subsection{Catalytic activity of Au NPs}

Aromatic amino compounds are widely used in industry as an intermediate in the preparation of polymers, azo dyes, etc. However, the chemical reduction of aromatic nitro compounds with sodium borohydride is extremely slow, and the use of a catalyst is essential. Of late, metal nanoparticles have been explored for this reaction. In this the reduction of nitro aromatics to its corresponding amino derivatives with an excess amount of $\mathrm{NaBH}_{4}$ has frequently been used as a model reaction to examine the catalytic performance of metal nanoparticles $[23,24]$.

It is often discussed in the literature that the presence of stabilizer can inhibit the catalytic activity of the metal nanoparticles [2]. In the present work it was possible to stabilize small Au NPs in the absence of PVP in 20:80 (v/v) glycerol:water mixture. Hence, for catalytic reaction $\mathrm{Au}$ NPs prepared in 20:80 (v/v) glycerol:water mixture were used; however, the catalytic reaction was carried out in an aqueous solution. The strong UV-Vis peaks

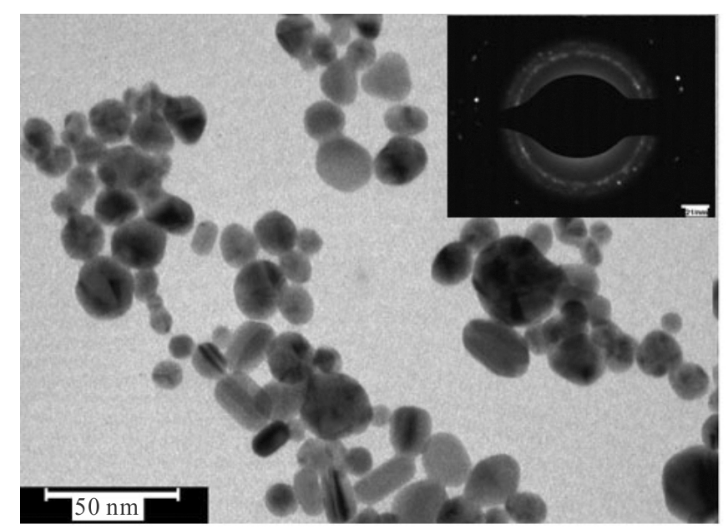

(a)

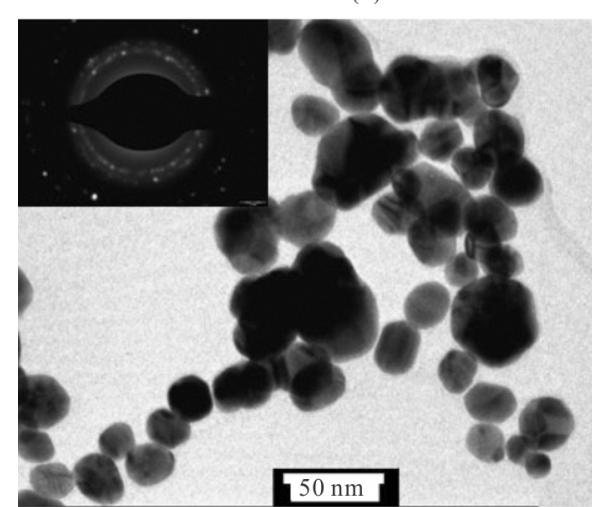

(b)

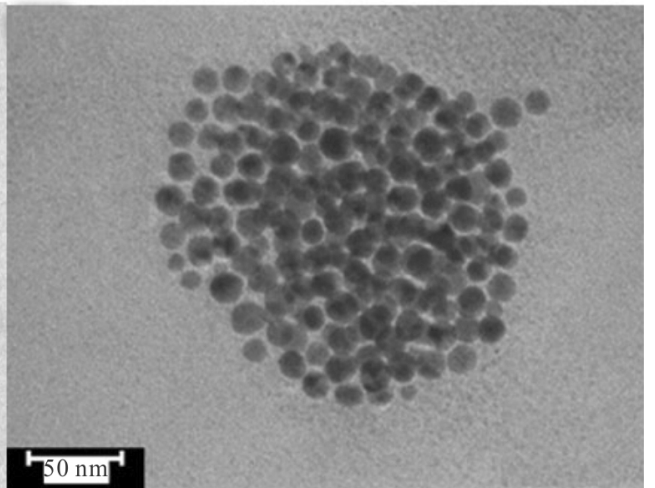

(c)

Figure 8. TEM images of Au NPs obtained in presence of different concentration of glycerol (a) $80 \%$ (b) $60 \%$ and (c) $20 \%$. 
Table 1. Summary of particle size and shape of Au NPs at different glycerol:water ratios (v/v) in the absence of PVP.

\begin{tabular}{cclcc}
\hline \% conc. of glycerol & SPR peak $(\mathrm{nm})$ & Shape and size distribution & Particle size by TEM (nm) & Colloidal stability \\
\hline $100 \%$ & 554 & $\begin{array}{l}\text { Non spherical, Polygonal } \\
\text { and polydispersed }\end{array}$ & 50 & Turbid after 1 day \\
$80 \%$ & 543 & $\begin{array}{l}\text { Non spherical, polygonal } \\
\text { and polydispersed }\end{array}$ & 25 & $\begin{array}{l}\text { Stable at least up to } 8 \\
\text { months }\end{array}$ \\
$50 \%$ & 533 & $\begin{array}{l}\text { Spherical and nearly } \\
\text { monodispersed }\end{array}$ & 20 & $\begin{array}{l}\text { Stable at least up to } 8 \\
\text { months }\end{array}$ \\
$20 \%$ & 522 & $\begin{array}{l}\text { Spherical and } \\
\text { monodispersed }\end{array}$ & 8 & $\begin{array}{l}\text { Stable at least up to } 8 \\
\text { months }\end{array}$ \\
\hline
\end{tabular}

Table 2. Variation in the rate of reduction of o-nitro aniline with increasing amount of Au NPs.

\begin{tabular}{ccc}
\hline Sr. No. & Concentration of $\mathrm{Au} \mathrm{NPs}(\mu \mathrm{g} / \mathrm{mL})$ & $\mathrm{k}_{\mathrm{obs}, \mathrm{s}} \mathrm{s}^{-1}$ \\
\hline 1 & 14 & $2.5 \times 10^{-3}$ \\
2 & 27 & $3.2 \times 10^{-3}$ \\
3 & 54 & $7.5 \times 10^{-3}$ \\
4 & 82 & $3 \times 10^{-2}$ \\
\hline
\end{tabular}

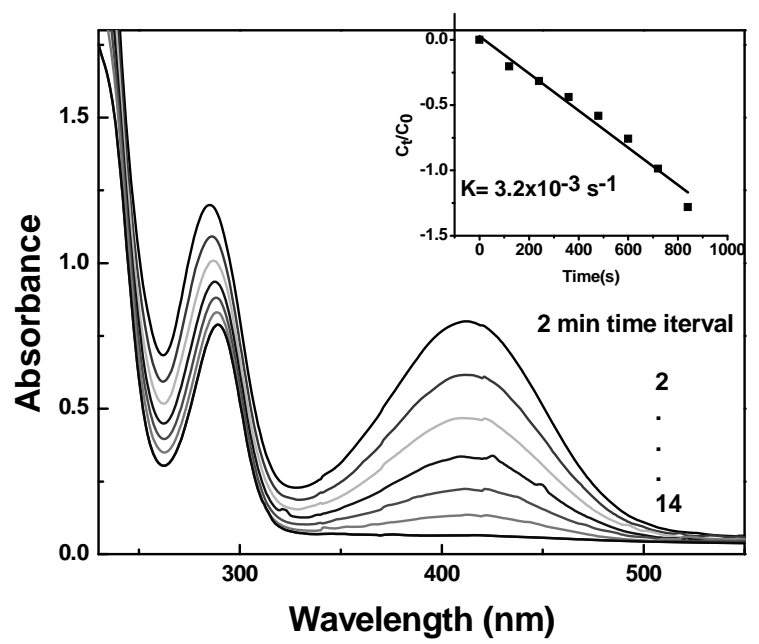

Figure 9. Time-dependent variation in the absorption spectrum of o-nitro aniline reduced by $5 \mu \mathrm{L} / \mathrm{mL}$ of Au NPs prepared in $20 \%$ glycerol. Inset: Plot of $\ln \left(C_{t} / C_{0}\right)$ versus time.

characteristic of o-nitro aniline appears at $412 \mathrm{~nm}$ and $283 \mathrm{~nm}$ [25]. It was found that there was a very slow decrease of absorbance during the chemical reaction without catalyst. However, after the addition of Au nanoparticles a decrease in the absorbance at $412 \mathrm{~nm}$ and shift of the peak from $283 \mathrm{~nm}$ to $289 \mathrm{~nm}$ with reaction time usually indicate steady reduction of o-nitro aniline to benzenediamine (Figure 9). The progress of reduction reaction was monitored by measuring UV-Vis absorption recorded at different times; $t$, at $298 \mathrm{~K}$ at different concentration of $\mathrm{Au}$ NPs. The ratio of $\mathrm{C}_{t}$ and $\mathrm{C}_{0}$, where $\mathrm{C}_{\mathrm{t}}$ and $\mathrm{C}_{0}$ refer to the concentration of o-nitro aniline at times $t$ and 0 , is measured from the relative intensity ratio of the respective absorbance, at $412 \mathrm{~nm}$. The linear correlation of $\ln \left(\mathrm{C}_{t} / \mathrm{C}_{0}\right)$ versus time is shown in inset of Figure 9 indicating the reaction follows pseudo first-order kinetics. The rate constant $(k)$ for the reaction, directly calculated from the slope of the straight line, for different concentration of Au NPs are tabulated in Table 2. It was found that when the gold content of the dispersion (ranging from 14 to $82 \mu \mathrm{g} / \mathrm{mL}$ ) was varied keeping other parameters constant, to determine the effect of the amount of catalyst on the rate of the reaction, the rate constant showed an increase of factor $c a$. 10 times.

\section{Conclusion}

Green synthesis of Au NPs was carried out by simply adding $\mathrm{NaOH}$ to the tetra chloroauric acid solution prepared in glycerol. Glycerol is used both as a reducing and stabilizing agent. The different size and shape of Au NPs can be prepared by varying the concentration of glycerol, concentration of $\mathrm{NaOH}$ and concentration of gold ions. Generally, stabilizers are added in the synthesis of the nanoparticles to prevent the aggregation of particles. The results of the present study show that the viscosity of the medium can play a similar role as of stabilizer by decreasing the interaction of the particles. However, there is no direct relationship between the viscosity and the stability of the particles. Thus the present work highlights that by exploiting the intrinsic properties of the solution it is possible to synthesize small and stable nanoparticles without using stabilizer. The Au NPs as synthesized in 20:80 (v/v) glycerol:water mixture were found to be catalytically active and had shown good catalytic activity for reduction of o-nitro aniline. 


\section{REFERENCES}

[1] H. Xiaohua, I. H. El-Sayed, W. Qian and M. A. El-Sayed, "Cancer Cell Imaging and Photothermal Therapy in the Near-Infrared Region by Using Gold Nanorods," Journal of American Chemical Society, Vol. 128, No. 6, 2006, pp. 2115-2120. doi:10.1021/ja057254a

[2] M. C. Daniel and D. Astruc, "Gold Nanoparticles: Assembly, Supramolecular Chemistry, Quantum-Size-Related Properties, and Applications toward Biology, Catalysis, and Nanotechnology," Chemical Reviews, Vol. 104, No. 1, 2004, pp. 293-346. doi:10.1021/cr030698+

[3] V. Tjoa, W. Jun, V. Dravid, S. Mhaisalkarad and N. Mathews, "Hybrid Graphene-Metal Nanoparticle Systems: Electronic Properties and Gas Interaction," Journal of Materials Chemistry, Vol. 21, No. 39, 2011, pp. 1559315599. doi: $10.1039 / \mathrm{c} 1 \mathrm{jm} 12676 \mathrm{~h}$

[4] L. Dykmana and N. Khlebtsov, "Gold Nanoparticles in Biomedical Applications: Recent Advances and Perspectives," Chemical Society Reviews, Vol. 41, No. 6, 2012, pp. 2256-2282. doi:10.1039/c1cs15166e

[5] B. R. Cuenya, "Synthesis and Catalytic Properties of Metal Nanoparticles: Size, Shape, Support, Composition, and Oxidation State Effects," Thin Solid Films, Vol. 518, No. 12, 2010, pp. 3127-3150. doi:10.1016/j.tsf.2010.01.018

[6] L. Rodriguez-Lorenzo, R. Rica, R. A. Alvarez-Puebla, L. M. Liz-Marzan and M. M. Stevens, "Plasmonic Nanosensors with Inverse Sensitivity by Means of Enzymeguided Crystal Growth," Nature Materials, Vol. 11, No. 7, 2012, pp. 604-607. doi:10.1038/nmat3337

[7] S. Y. Li and M. Wang, "Branched Metal Nanoparticles: A Review on Wet-Chemical Synthesis and Biomedical Applications," Nano Life, Vol. 2, No. 1, 2012, Article ID: 1230002. doi:10.1142/S1793984411000311

[8] S. Kapoor, R. Joshi and T. Mukherjee, "Influence of IAnions on the Formation and Stabilization of Copper Nanoparticles," Chemical Physics Letters, Vol. 351, No. 5-6, 2002, pp. 443-448. doi:10.1016/S0009-2614(02)00159-8

[9] M. Vaseem, K. M. Lee, D. Y. Kim and Y. -B. Hahn, "Parametric Study of Cost-Effective Synthesis of Crystalline Copper Nanoparticles and their Crystallographic Characterization," Material Chemistry and Physics, Vol. 125, No. 3, 2011, pp 334-34. doi:10.1016/j.matchemphys.2010.11.007

[10] K. S. N. Kamarudin and M. F. Mohamad, "Synthesis of Gold (Au) Nanoparticles for Mercury Adsorption," American Journal of Applied Sciences, Vol. 7, No. 6, 2010, pp. 835-839. doi:10.3844/ajassp.2010.835.839

[11] M. S. Frank, M. C. Nahata and M. D. Hilty, "Glycerol: A Review of Its Pharmacology, Pharmacokinetics, Adverse Reactions, and Clinical Use," Pharmacotherapy, Vol. 1, No. 2, 1981, pp. 147-160.

[12] H. W. Felter, "Glycerinum," 1922. http://www.henriettesherbal.com/eclectic/felter/glycerinu m.html

[13] R. Genc, G. Clergeaud, M. Ortiz and C. K. O'Sullivan, "Green Synthesis of Gold Nanoparticles Using Glycerol-
Incorporated Nanosized Liposomes," Langmuir, Vol. 27, No. 17, 2011, pp. 10894-10900.

doi:10.1021/la201771s

[14] K. Singh, K. H. Kate1, V. V. S. Chilukuri and P. K. Khanna, "Glycerol Mediated Low Temperature Synthesis of Nickel Nanoparticles by Solution Reduction Method," Journal of Nanoscience and Nanotechnology, Vol. 11, No. 6, 2011, pp. 5131-5136.

doi:10.1166/jnn.2011.4142

[15] E. Nisaratanaporn and K. Wongsuwan, "Preparation of Ultrafine Silver Powder Using Glycerol as Reducing Agent," Journal of Metals, Materials and Minerals, Vol. 18, No. 2, 2008, pp. 1-5.

[16] N. Grace and K. Pandian, "One Pot Synthesis of Polymer Protected Gold Nanoparticles and Nanoprisms in Glycerol," Colloids and Surfaces A: Physicochemical and Engineering Aspects, Vol. 290, No. 1-3, 2006, pp. 138-142. doi:10.1016/j.colsurfa.2006.05.015

[17] V. Sharma, K. Park and M. Srinivasarao, "Colloidal Dispersion of Gold Nanorods: Historical Background, Optical Properties, Seed-Mediated Synthesis, Shape Separation and Self-Assembly," Materials Science and Engineering: R: Reports, Vol. 65, No. 1-3, 2009, pp. 1-38. doi:10.1016/j.mser.2009.02.002

[18] S. Yang, T. Zhang, L. Zhang, S. Wang, Z. Yang and B. Ding, "Continuous Synthesis of Gold Nanoparticles and Nanoplates with Controlled Size and Shape under UV Irradiation," Colloids and Surfaces A: Physicochemical and Engineering Aspects, Vol. 296, No. 1-3, 2007, pp. 37-44. doi:10.1016/j.colsurfa.2006.09.017

[19] S. Ghosh, S. Patil, M. Ahire, R. Kitture, D. D. Gurav, A. M. Jabgunde, S. Kale, K. Pardesi, V. Shinde, J. Bellare, D. D. Dhavale and B. A. Chopade, "Gnidia Glauca Flower Extract Mediated Synthesis of Gold Nanoparticles and Evaluation of its Chemocatalytic Potential," International Journal of Nanobiotechnology, Vol. 10, No. 1, 2012, pp. 17-26.

[20] L. Chen, C. Wan and Y. Wang, "Chemical Preparation of Pd Nanoparticles in Room Temperature Ethylene Glycol System and Its Application to Electroless Copper Deposition," Journal of Colloid and Interface Science, Vol. 297, No. 1, 297, 2006, pp. 143-150. doi:10.1016/j.jcis.2005.10.029

[21] S. Boopathi, S. Senthilkumar and K. L. Phani, "Facile and One Pot Synthesis of Gold Nanoparticles Using Tetraphenylborate and Polyvinylpyrrolidone for Selective Colorimetric Detection of Mercury Ions in Aqueous Medium," Journal of Analytical Methods in Chemistry, Vol. 2012, No. 1, 2012, Article ID: 348965. doi: $10.1155 / 2012 / 348965$

[22] G. Oza, S. Pandey, R. Shah, M. Vishwanathan, R. Kesarkar, M. Sharon and M. Sharon, "Tailoring Aspect Ratio of Gold Nano Rods: Impact of Temperature, $\mathrm{pH}$, Silver ions, CTAB Concentration and Centrifugation," Advances in Applied Science Research, Vol. 3, No. 2, 2012, pp. 1027-1038.

[23] K. Layek, M. Lakshmi Kantam, M. Shirai, D. NishioHamane, T. Sasaki and H. Maheswaran, "Gold Nanoparticles Stabilized on Nanocrystalline Magnesium Oxide as 
an Active Catalyst for Reduction of Nitroarenes in Aqueous Medium at Room Temperature," Green Chemistry, Vol. 14, No. 11, 2012, pp. 3164-3174. doi:10.1039/c2gc35917k

[24] A. Leelavathi, T. U Bhaskara Rao and T. Pradeep, "Supported Quantum Clusters of Silver as Enhanced Catalysts for Reduction," Nanoscale Research Letters, Vol. 6, No. 2, 2011, pp. 123-132. doi:10.1186/1556-276X-6-123

[25] N. Pradhan, A. Pal and T. Pal, "Catalytic Reduction of Aromatic Nitro Compounds by Coinage Metal Nanoparticles," Langmuir, Vol. 17, No. 5, 2001, pp 1800-1802. doi:10.1021/la000862d 